\title{
Propriedades biológicas de Rhaphiodon echinus: uma breve revisão da literatura
}

\author{
Biological properties of Rhaphiodon echinus: a brief literature review \\ Propiedades biológicas de Rhaphiodon echinus: una breve revisión de la literatura
}

Júlia Kiara da Nóbrega Holanda ORCID: https://orcid.org/0000-0002-1426-0714 Universidade Federal de Campina Grande, Brasil E-mail: juliakiaranh@gmail.com Lívia da Silva Pereira ORCID: https://orcid.org/0000-0003-2057-3570 Universidade Federal de Campina Grande, Brasil E-mail: liviadasilva9990@gmail.com

Wanessa Medeiros da Silva ORCID: https://orcid.org/0000-0003-2881-2724 Universidade Federal de Campina Grande, Brasil E-mail: wanessamds01@gmail.com

Ana Beatriz Bomfim Gomes Ribeiro ORCID: https://orcid.org/0000-0001-9069-2386 Universidade Federal de Campina Grande, Brasil E-mail: abeatrizbomfim98@gmail.com

Samara Crislâny Araújo de Sousa ORCID: https://orcid.org/0000-0003-4911-3797 Universidade Federal de Campina Grande, Brasil E-mail: samaracrislany06@gmail.com

Viton Dyrk Guimarães Fernandes ORCID: https://orcid.org/0000-0003-4901-7391 Universidade Federal de Campina Grande, Brasil E-mail: vitondyrk@gmail.com

Raline Mendonça dos Anjos ORCID: https://orcid.org/0000-0003-0751-7523 Universidade Federal de Campina Grande, Brasil

E-mail: raline.anjos@gmail.com Maria Angélica Sátyro Gomes Alves ORCID: https://orcid.org/0000-0003-3329-8360 Universidade Federal de Campina Grande, Brasil E-mail: angelicasatyro@hotmail.com

Gymenna Maria Tenório Guênes ORCID: https://orcid.org/0000-0002-5447-0193 Universidade Federal de Campina Grande, Brasil E-mail: gymennat@yahoo.com.br

Abrahão Alves de Oliveira Filho ORCID: https://orcid.org/0000-0002-7466-9933 Universidade Federal de Campina Grande, Brasil E-mail: abrahao.farm@gmail.com

\begin{abstract}
Resumo
O Brasil ocupa o primeiro lugar do mundo em biodiversidade terrestre, abrigando cerca de 200.000 espécies vegetais nativas, as quais avalia-se que ao menos metade possua alguma propriedade terapêutica. Sob essa perspectiva, na atual conjuntura, as terapias alternativas naturais têm tido um panorama crescente, visto os diversos benefícios desta prática, tais como menor toxicidade, maior biocompatibilidade e custo mais acessível. Dentre as diversas espécies vegetais existentes, a Rhaphiodon echinus, erva endêmica e nativa do Brasil incluída na família Lamiaceae, se destaca pelo desempenho em atividades farmacológicas. Diante disso, o objetivo desta revisão é discorrer acerca das propriedades etnobotânicas, fitoquímicas e farmacológicas desta espécie vegetal. Para isso, realizou-se uma busca da literatura publicada entre 2011 e 2021 nos bancos de dados on-line National Libary of Medicine (PubMed), Google Acadêmico, Repositório Institucional da UNESP; Hoehnea- Instituto de Botânica; Acervo Digital da UFPR; Revista Arquivo Cientifico e Pharmaceutical Biology. Após a análise dos dados encontrados na literatura, seguindo os critérios de inclusão estabelecidos, verificou-se que a Rhaphiodon echinus é uma espécie promissora para a indústria de medicamentos, visto que apresenta ação fotoprotetora, atividade antifúngica contra cepas de Candida tropicalis, além de potencial antioxidante, anti-inflamatório e antifúngico quando usado no tratamento de candidíase oral e distúrbios no trato digestivo. Ainda, quando associada à antimicrobianos contra cepas de algumas espécies fúngicas e bacterianas, a Rhaphiodon echinus apresenta atividade anticabteriana e antifúngica mais elevada, sugerindo efeito
\end{abstract}


sinérgico. No entanto, mais estudos metodologicamente estruturados precisam ser realizados para melhor elucidar seu amplo potencial terapêutico.

Palavras-chave: Rhaphiodon echinus; Fitoquímica; Botânica; Atividade farmacológica.

\begin{abstract}
Brazil occupies the first place in the world in terrestrial biodiversity, sheltering about 200,000 native plant species, which it is estimated that at least half have some therapeutic property. From this perspective, in the current situation, natural alternative therapies have had a growing panorama, given the various benefits of this practice, such as less toxicity, greater biocompatibility and more affordable cost. Among the various existing plant species, Rhaphiodon echinus, an endemic herb native to Brazil included in the Lamiaceae family, stands out for its performance in pharmacological activities. Therefore, the objective of this review is to discuss the ethnobotanical, phytochemical and pharmacological properties of this plant species. For this, a search of the literature published between 2011 and 2021 in the online databases National Libary of Medicine (PubMed), Academic Google, Institutional Repository of UNESP; Hoehnea- Institute of Botany; UFPR's Digital Collection; Scientific Archive and Pharmaceutical Biology Magazine. After analyzing the data found in the literature, following the established inclusion criteria, it was found that Rhaphiodon echinus is a promising species for the drug industry, as it has photoprotective action, antifungal activity against Candida tropicalis strains, in addition to potential antioxidant, anti-inflammatory and antifungal when used to treat oral thrush and digestive tract disorders. Also, when associated with antimicrobials against strains of some fungal and bacterial species, Rhaphiodon echinus has higher anti-cabterian and antifungal activity, suggesting a synergistic effect. However, more methodologically structured studies need to be carried out to better elucidate its broad therapeutic potential.
\end{abstract}

Keywords: Rhaphiodon echinus; Phytochemistry; Botany; Pharmacological activity.

\title{
Resumen
}

Brasil ocupa el primer lugar en el mundo en términos de biodiversidad terrestre, albergando alrededor de 200.000 especies de plantas nativas, de las cuales se estima que al menos la mitad tiene alguna propiedad terapéutica. Desde esta perspectiva, en la coyuntura actual, las terapias alternativas naturales han tenido un panorama creciente, dado los diversos beneficios de esta práctica, como menor toxicidad, mayor biocompatibilidad y costo más accesible. Entre las varias especies de plantas existentes, Rhaphiodon echinus, una hierba endémica y nativa de Brasil incluida en la familia Lamiaceae, se destaca por su desempeño en actividades farmacológicas. Por lo tanto, el objetivo de esta revisión es discutir las propiedades etnobotánicas, fitoquímicas y farmacológicas de esta especie vegetal. Para ello, se realizó una búsqueda de la literatura publicada entre 2011 y 2021 en las bases de datos en línea Biblioteca Nacional de Medicina (PubMed), Google Scholar, Repositorio Institucional de la UNESP; Hoehnea-Instituto de Botánica; Colección Digital UFPR; Archivo Científico y Revista de Biología Farmacéutica. Luego de analizar los datos encontrados en la literatura, siguiendo los criterios de inclusión establecidos, se encontró que Rhaphiodon echinus es una especie promisoria para la industria farmacéutica, ya que posee acción fotoprotectora, actividad antifúngica contra cepas de Candida tropicalis, además de potencial antioxidante, antiinflamatorio y antifúngico cuando se usa en el tratamiento de la candidiasis oral y trastornos en el tracto digestivo. Además, cuando se asocia con antimicrobianos contra cepas de algunas especies fúngicas y bacterianas, Rhaphiodon echinus muestra una mayor actividad anticabteriana y antifúngica, lo que sugiere un efecto sinérgico. Sin embargo, es necesario realizar estudios más estructurados metodológicamente para dilucidar mejor su amplio potencial terapéutico.

Palabras clave: Rhaphiodon echinus; Fitoquímica; Botánica; Actividad farmacológica.

\section{Introdução}

A fitoterapia é designada pelo uso externo ou interno de plantas medicinais, em suas diferentes formas farmacêuticas, in natura ou como medicamento, com finalidade terapêutica (Silva et al., 2017; Monteiro \& Fraga, 2021). Sua aplicação possui origens antigas, sendo comumente aplicada na população até meados do século XX, quando no período pós-guerra, com o advento de novas tecnologias, passou-se a utilizar medicamentos sintéticos e alopáticos. Na atual conjuntura, as terapias alternativas naturais têm tido um panorama crescente, visto os diversos benefícios desta prática como menor toxicidade, maior biocompatibilidade e custo mais acessível (Espírito Santo et al., 2020; Matsuchita \& Matsuchita, 2015).

A utilização de plantas medicinais se dá por meio de diferentes partes do vegetal, como raiz, caule, semente, folha e fruto, os quais podem prover substâncias bioativas, os metabólitos secundários, que manifestam diversas propriedades biológicas, farmacêuticas e terapêuticas (Santos et al., 2021). Dentre esses metabólitos apresentam-se os compostos fenólicos, terpenos e alcalóides, capazes de desempenhar diversas atividades curativas, dentre elas a capacidade cicatrizante, antiinflamatória, anti-parasitária e anti-hipertensiva (Fernandes et al., 2019; Guimarães et al., 2021). 
Nesse contexto, o Brasil ocupa o primeiro lugar do mundo em biodiversidade terrestre, abrigando cerca de 200.000 espécies vegetais nativas, as quais avalia-se que ao menos metade possua alguma propriedade terapêtica, e apenas $1 \%$ das espécies potenciais foram alvo de estudos. Nesse sentido, as plantas oferecem um recurso renovável na descoberta de novas biomoléculas terapêuticas, devido às características estruturais e biológicas de seus diferentes constituintes (De Rossi et al., 2021; Guimarães et al., 2021; Santos et al., 2019).

Com efeito, a espécie de planta medicinal Rhaphiodon echinus pertencente à família botânica Lamiaceae e conhecida popularmente como "betônica", destaca-se como uma espécie endêmica do bioma caatinga e, portanto, encontra-se amplamente distribuída na região Nordeste do Brasil (Neto et al., 2021). Com apresentação global, a família Lamiaceae detém aproximadamente 240 gêneros e 7200 espécies com ocorrência desde regiões tropicais até áreas de clima temperado. No Brasil encontram-se 32 gêneros e 496 espécies dessa família, com relevância para o gênero Hyptis e Rhaphiodon presente no semiárido brasileiro (Ferreira et al., 2019).

Por conseguinte, diversas pesquisas farmacológicas que abordam a espécie $R$. echinus como objeto de estudo, indicam seu potencial para atividades biológicas antimicrobiana e antioxidante, exibindo propriedades anti-inflamatórias e analgésicas atribuídas à compostos oriundos do metabolismo vegetal (Duarte et al., 2016).

Ademais, Costa et al. (2017) avaliaram a composição química dos extratos aquoso e etanólico de R. echinus, e evidenciaram que o ácido clorogênico e o ácido elágico foram os principais constituintes dos extratos. Ainda foi possível verificar, através de ensaios in vitro, o papel modulador dos extratos sendo eficaz no aumento do efeito antibiótico da nistatina e do fluconazol contra a cepa multirresistente de C. krusei, de modo a demonstrar a capacidade dos extratos de R. echinus em potencializar a atividade de medicamentos antifúngicos.

Diante disso, embasado na literatura científica e nas informações expressadas à utilização da fitoterapia, o presente estudo objetiva, por meio de uma revisão narrativa, discorrer acerca das propriedades etnobotânicas, fitoquímicas e farmacológicas da espécie vegetal Rhaphiodon echinus.

\section{Metodologia}

O presente estudo trata-se de uma revisão narrativa da literatura, realizada com dados bibliográficos de 2011 a 2021 publicados nos bancos de dados on-line National Libary of Medicine (PubMed), Google Acadêmico, Repositório Institucional da UNESP; Hoehnea- Instituto de Botânica; Acervo Digital da UFPR; Revista Arquivo Cientifico e Pharmaceutical Biology. Para isso, foram utilizados quatro campos semânticos: "Rhaphiodon echinus" em associação com "Fitoquímica", "Botânica" e "Atividade farmacológica".

De acordo com Moreira (2004), a revisão de literatura é classificada como uma obra que visa analisar a produção bibliográfica em determinada área temática, em um espaço de tempo, proporcionando uma visão geral sobre um assunto específico, ressaltando novas ideias e técnicas que têm recebido determinada ênfase na literatura escolhida.

Para a seleção dos artigos não foi imposta limitação de idioma, além disso, outros critérios de inclusão foram: (1) revisões sistemáticas da literatura; dissertações, teses e pesquisas laboratoriais; (2) trabalhos científicos publicados que informassem dados pertinentes e relevantes sobre os diversos aspectos, funções e propriedades fitoterápicas desempenhadas pelo Rhaphiodon echinus. Foram excluídos da pesquisa: (1) artigos científicos publicados fora do intervalo de tempo estabelecido e (2) estudos que não se encaixassem ao objetivo proposto.

Inicialmente, a partir da leitura do título e resumo, foram escolhidos os manuscritos potencialmente elegíveis. Os estudos que se adequavam a temática foram selecionados para uma leitura completa e, considerando os critérios propostos, foram coletadas algumas informações que posteriormente foram analisadas e categorizadas de forma descritiva/narrativa neste estudo. 


\section{Resultados e Discussão}

\subsection{Características botânicas}

A Rhaphiodon echinus é uma erva endêmica e nativa do Brasil incluída na família Lamiaceae e a única espécie descrita do gênero Rhaphiodon. Tem ocorrências registradas nas regiões Sudeste, Centro-oeste e Nordeste, tendo uma distribuição mais evidente no Nordeste Brasileiro e podendo ser encontrada nos biomas da Caatinga, Cerrado e Mata Atlântica (Flora do Brasil, 2021).

Na sua classificação morfológica ela é dita como uma erva prostrada que apresenta um conjunto de sépalas frutíferas, lacínios espinescentes, flores de cor violeta reunidas em glomérulos auxiliares e terminais, cálice possuindo lobos espinescentes e sua corola apresenta a colocação roxa (Alves, 2019; Flora do Brasil, 2021).

\subsection{Avaliação fitoquímica}

As espécies da família Lamiaceae são conhecidas pela sua grande ocorrência em todo o mundo e a grande utilização dos seus componentes na culinária, o que levou à varias pesquisas científicas e publicações literárias sobre os indivíduos que incorporam esse táxon (Lima \& Cardoso, 2013) A espécie Rhaphiodon echinus apresenta metabolitos secundários como taninos, flavonoides e saponinas (Medeiros et al., 2021a) e é conhecida por apresentar grande potencial para uso terapêutico na crença popular. No trabalho de Neto et al. (2021), foram realizados experimentos laboratoriais utilizando seu óleo essencial e extrato de folhas com o intuito de confirmar seu poder medicinal; esses experimentos levaram à validação científica de seu potencial antioxidante, anti-inflamatório e antifúngico quando usado no tratamento de candidíase oral e distúrbios no trato digestivo.

Segundo Duarte (2016), o óleo essencial do Rhaphiodon echinus pode ser indicado no combate de doenças neurogenoativas e infecciosas devido à sua atividade quelante ao ferro (II). Em sua composição química também foram encontrados monoterpenos e sesquiterpenos, substâncias importantes no aumento da atividade antibacteriana e antifúngica, indicando que seu óleo seria promissor no desenvolvimento de medicamentos antimicrobianos.

\subsection{Estudos biológicos}

A Rhaphiodon echinus apresenta atividade antifúngica, observada no estudo de Ferreira et. al. (2019), que usou os extratos aquoso e alcóolico das partes aéreas dessa espécie vegetal contra cepas de Candida tropicalis, apresentando uma forte capacidade antifúngica.

Além disso, Duarte et. al. (2016), realizou um estudo para avaliar a capacidade antibacteriana do óleo essencial de Rhaphiodon echinus associado a antimicrobianos contra cepas fúngicas de C. albicans, C. krusei, C. tropicalis e cepas bacterianas de E. coli, S. aureus e Pseudomonas. Como resultado obteve-se uma atividade antimicrobiana e antifúngica baixa, porém se tornou alta quando associada aos antimicrobianos, sugerindo um efeito sinérgico. Esse efeito pode se dar devido a presença de monoterpenos e sesquiterpenos.

Medeiros et al. (2020), em estudo que buscou avaliar, in vitro, a atividade fotoprotetora do extrato etanólico de Rhaphiodon (R.) echinus, com diferentes concentrações do produto natural $(50,100,500$ e $1000 \mu \mathrm{g} / \mathrm{mL})$, através de varreduras com o auxílio de um espectrofotrometro, nas faixas (290 a $320 \mathrm{~nm}$ ) com intervalos de $5 \mathrm{~nm}$, obteve que apenas as duas maiores concentrações $(500 \mu \mathrm{g} / \mathrm{mL}$ e $1000 \mu \mathrm{g} / \mathrm{mL})$ apresentaram valores de fotoproteção consideráveis e acima do fator mínimo de proteção de 6 (FPS), sendo 23,65 e 25,00 respectivamente. Assim, a atividade fotoprotetora do extrato de R. echinus demonstra importante característica bioativa.

Ao realizar a avaliação da atividade fotoprotetora in vitro do extrato aquoso de Rhaphiodon echinus (Nees \& Mart.) Schauer, em diferentes concentrações $(50,100,500$ e $1000 \mu \mathrm{g} / \mathrm{mL})$ e realizado varreduras em espectrofotômetro no 
comprimento de onda de 290 a $320 \mathrm{~nm}$ com intervalos de $5 \mathrm{~nm}$ a cada 5 minutos, Medeiros et al. (2021a) observou que produto apresentou fotoproteção em todas as concentrações avaliadas (>6FPS), sendo as concentrações de 500 a $1000 \mu \mathrm{g} / \mathrm{mL}$ as que obtiveram maior efeito fotoprotetor com >25FPS.

Em outro estudo, Medeiros et al. (2021b), ao avaliar o potencial fotoprotetor, in vitro, do extrato fase acetato de $R$. echinus, obteve que as concentrações de $(50,100,500$ e 1000) $\mu \mathrm{g} / \mathrm{mL}-1$ apresentaram valores de fotoproteção igual à 9,47 , 25,00, 25,00 e 25,00 (FPS), respectivamente, comprovando-se a atividade fotoprotetora, in vitro, do extrato fase acetato de $R$. echinus.

Costa et al. (2017) investigaram pela primeira vez a atividade antimicrobiana (antifúngica e antibacteriana) de extratos etanólicos e aquosos de R. echinus contra cepas multirresistentes de bactérias (E. coli, P. aeruginosa e $S$. aureus) e fungos ( $C$. albicans, $C$. krusei e C. tropicalis), bem como potencial para aumentar a atividade de antibióticos. Ademais, ambos os extratos foram quimicamente caracterizados e sua toxicidade foi avaliada em Artemia salina. Com isso, os resultados demonstraram que o extrato aquoso de R. echinus causou um aumento significativo na atividade dos antibióticos gentamicina e imipenem, enquanto o extrato etanólico aumentou fortemente a atividade antibiótica da gentamicina, amicacina, imipenem e ciprofloxacina contra $P$. aeruginosa. No entanto, nenhum dos extratos afetaram significativamente a atividade antibiótica das drogas quando testadas contra S. aureus. A análise fitoquímica dos extratos indicou ácido elágico, ácido caféico e ácido clorogênico como os principais componentes que podem ser, pelo menos em parte, responsáveis pelo aumento da atividade dos antibióticos. Nenhum dos extratos apresentou toxicidade em A. salina mesmo na maior concentração testada (1000 $\mu \mathrm{g} / \mathrm{mL})$.

Pio et. al (2019), ao realizar um levantamento etnobotânico de plantas medicinais utilizadas em ilhas do submédio do rio São Francisco, conforme a indicação da comunidade, obteve que a espécie Rhaphiodon echinus (Ness \& Mart.) Schauer foi a mais citada, com principal indicação para tratar infecção do trato urinário e dismenorreia. Posteriormente, ao realizar investigação fitoquímica e farmacológica utilizando partes aéreas da espécie, revelou prevalência de flavonoides, taninos, lignanas e saponinas no produto liofilizado e extrato acetato de etila, bem como compostos terpênicos no extrato hexânico e clorofórmico. Os testes antibacterianos mostraram que o extrato acetato de etila é mais atuante contra E. coli e S. aureus do que para $P$. aeruginosa; o extrato etanólico bruto tem efeito parcial contra bactérias gram-negativas e o óleo essencial possui moderada resposta para todos os microorganismos testados. O produto liofilizado, o extrato etanólico bruto e o extrato hexânico possuem atividade espasmolítica dependente da concentração, sem diferença significativa e sem relaxamento total.

Em estudo conduzido por Duarte et al. (2016), o óleo essencial da folha de R. echinus foi investigado por suas propriedades antimicrobianas, determinada pelo método de microdiluição, considerando o efeito do óleo sozinho, e em combinação com antifúngicos ou antibióticos contra Candida albicans, Candida krusei, Candida tropicalis e Escherichia coli, Staphylococcus aureus e Pseudomonas. Além disso, foi determinado seu potencial de quelação do ferro (II). Ao ser caracterizado por cromatografia gasosa-espectrometria de massa, o óleo essencial de $R$. echinus revelou a presença de monoterpenos e sesquiterpenos. Os resultados mostraram a presença de $\beta$-cariofileno e biciclogermacreno nos compostos, e revelaram baixa atividade antifúngica e antibacteriana do óleo essencial, mas forte efeito modulador sobre os antimicrobianos quando associados ao óleo. O óleo essencial apresentou atividade quelante de ferro (II). Dessa forma, sugere-se que o óleo essencial de $R$. echinus é um produto natural capaz de aumentar a atividade antibacteriana e antifúngica de drogas antimicrobianas.

\section{Conclusão}

Após a análise dos dados encontrados na literatura, pode-se concluir que a Rhaphiodon echinus é uma espécie promissora para o desenvolvimento de medicamentos, visto que estudos revelam ação fotoprotetora, atividade antifúngica contra cepas de Candida tropicalis, potencial antioxidante, anti-inflamatório e antifúngico quando usado no tratamento de 
candidíase oral e distúrbios no trato digestivo, bem como, pode ser indicada no combate de doenças neurogenoativas e infecciosas. Além disso, quando associada a antimicrobianos contra cepas fúngicas de C. albicans, C. krusei, C. tropicalis e cepas bacterianas de E. coli, S. aureus e Pseudomonas, a Rhaphiodon echinus, apresenta atividade antimicrobiana mais elevada, sugerindo efeito sinérgico.

No entanto, mais estudos metodologicamente estruturados precisam ser realizados para melhor elucidar seu amplo potencial terapêutico, mecanismos e padrões de eficiência e eficácia.

\section{Referências}

Alves, S. (2019). Tempo de florescimento da Rhaphiodon echinus schauer, em resposta a frequência de irrigação e densidade de plantio. [Dissertação de mestrado em Engenharia Agrícola, Universidade Federal do Vale do São Francisco]. Repositório UNIVASF.

Costa, A. R., Silva, J. L., Lima, K. R. R., Rocha, M. I., Barros, L. M., da Costa, J. G. M., Boligon, A. A., Kamdem, J. P., Carneiro, J. N. P., Leite, N. F., Menezes, I. R. A., Duarte, A. E., Morais-Braga, M. F. B. \& Coutinho, H. D. M. (2017). Rhaphiodon echinus (Nees \& Mart.) Schauer: Chemical, toxicological activity and increased antibiotic activity of antifungal drug activity and antibacterial. Microbial pathogenesis, 107(1), 280-286.

De Rossi, P. H. S, Giannoni, J. A., Oshiwa, M., Barbalho, S. M., Brunatti, A. C. S., Escouto, L. F. S., Sampaio, A. A. J., Pereira, M. M. \& Costa, T. B. (2021). Biodiversidade das Plantas Medicinais: benefícios e riscos. Revista PubSaúde, 5(139).

Duarte, A. E., de Menezes, I. R. A., Braga, M. F. B. M., Leite, N. F., Barros, L. M., Waczuk, E. P., da Silva, M. A. P., Boligon, A., Rocha, J. B. T., Souza, D. O., Kamdem, J. P., Coutinho, H. D. M. \& Burger, M. E. (2016). Antimicrobial activity and Modulatory effect of essential oil from the leaf of Rhaphiodon echinus (Nees \& Mart) Schauer on some antimicrobial drugs. Molecules, 21(6), 743.

Espírito Santo, R. P., Pereira, C. L., Detone, V. M. \& Chaves, M. G. A. M. (2020). Fitoterapia como alternativa à medicação intracanal convencional. Revista Fitos, 14(3), 382-389.

Fernandes, B. F., Gonçalves, H. R., Guimarães, M. R., Alves, A. A. \& Bieski, I. G. C. (2019). Estudo etnofarmacológico das plantas medicinais com presença de saponinas e sua importância medicinal. Revista da Saúde da AJES, 5(9).

Ferreira, J. L. S., Cavalcante, J. N. M., de Souza Siqueira, D., Bezerra, R. V., Delgado, L. A., de Oliveira, H. M. B. F., Vieira, E. S. S. V., Maia, G. L. A., Lima, E. O. L. \& Oliveira Filho, A. (2019). Comparação da atividade antifúngica do extrato aquoso e do extrato etanólico de Rhaphiodon echinus (Lamiaceae) contra cepas Candida tropicalis. Revista da Universidade Vale do Rio Verde, 17(1).

Guimarães, B. M., Ramos, K. A., de Souza, M. C., Franco, M. L., de Souza Alves, C. C., de Paula Carli, A. \& Coqueiro, J. M. (2021). Práticas terapêuticas com plantas medicinais para o tratamento do Diabetes Mellitus. Research, Society and Development, 10(10).

Lima, R. \& Cardoso, M. (2013). Família Lamiaceae: importantes óleos essenciais com ação biológica e antioxidante. Revista Fitos, 3(3), 14-24.

Matsuchita, H. L. P. \& Matsuchita, A. S. P. (2015). A Contextualização da Fitoterapia na Saúde Pública. Uniciências, 19(1).

Medeiros, M. A. C., Santos, B., Marques, F. M. C., Leite, M. F. M. S., Simões, M. M., Anjos, R. M., Júnior, L. B., Maia, G. L. A., Rosendo, R. A., Souza, A. P. \& Oliveira Filho, A. A. (2021b). Avaliação da atividade fotoprotetora do extrato fase acetato de Rhaphiodon echinus (Nees \& Mart.) Schauer. Scientific Electronic Archives, 14(8).

Medeiros, M. A. C., Simões, M. M., Santos, B., Marques, F. M. C., da Silva Leite, M. F. M., de Oliveira, H. M. B. F., de Oliveira, V. F., da Cunha, S. M. D., Maia, G. L. A., de Sousa, A. P. \& de Oliveira Filho, A. A. (2020). Avaliação da atividade fotoprotetora do extrato etanólico de Rhaphiodon echinus Schauer. Research, Society and Development, 9(7).

Medeiros, M. A. C., Santos, B., Marques, F. M. C., da Silva Leite, M. F. M., Simões, M. M., dos Anjos, R. M., Brito Júnior, L., Maia, G. L. A., Alves, M. A. S. G., de Sousa, A. P. \& de Oliveira Filho, A. A. (2021a). Avaliação da atividade fotoprotetora do extrato aquoso de Rhaphiodon echinus (Nees \& Mart.) Schauer. Scientia Plena, 17(4).

Monteiro, M. H. D. A. \& Fraga, S. A. P. M. (2021). Fitoterapia na prática clínica odontológica: produtos de origem vegetal e fitoterápicos. Revista Fitos, $15(1), 58-77$.

Moreira, W. (2004). Revisão de Literatura e Desenvolvimento Científico: conceitos e estratégias para confecção. 1(1): 19-31.

Mota, M. C. A. \& Pastore, J. F. B. (2020). Rhaphiodon in Flora do Brasil 2020. http://floradobrasil.jbrj.gov.br/reflora/floradobrasil/FB8295

Cruz Neto, J., Viana, J. W. M., Silva, V. B., Costa, A. R., Costa, M. H. N., Santos, M. A. F., Rodrigues, J. L. G., Teixeira, P. H. R., Oliveira, J. P. C., Correia, D. B., Menezes, S. A., Santos, L. T., Oliveira, M. G., Silva Junior, J. P., Gurgel, C. L., Sampaio, R. S. L., Lima, J. F., Araújo, N. J. S. \& Almeida-Bezerra, J. W. (2021). Revisão do uso medicinal de Rhaphiodon echinus Schauer (Lamiaceae): Atividades biológica e farmacológica. Research, Society and Development, 10(3), 01-12.

Pio, I. D. S. L., Lavor, A. L., Damasceno, C. M. D., Menezes, P. M. N., Silva, F. S. \& Maia, G. L. A. (2019). Conhecimento tradicional e usos de plantas medicinais por habitantes das ilhas do rio São Francisco, Brasil e análise preliminar de Rhaphiodon echinus (Lamiaceae). Brazilian Journal of Biology, 79(1), 87-99.

Santos, D. L., Moraes, J. S., de Souza Araújo, Z. T. \& da Silva, I. R. (2019). Saberes tradicionais sobre plantas medicinais na conservação da biodiversidade amazônica. Ciências em Foco, 12(1). 
Research, Society and Development, v. 11, n. 3, e20111326171, 2022

(CC BY 4.0) | ISSN 2525-3409 | DOI: http://dx.doi.org/10.33448/rsd-v11i3.26171

Santos, L., Fuzaro, C. C., Fracasso, J. A. R., Ibe Ibe, M. B., Parron, M. C. \& Rodrigues, M. M. A. (2021) Plantas Medicinais: suas associações e usos. Editora Científica. Produtos Naturais e Suas Aplicações da comunidade para o laboratório.

Silva, N. C. S., Vítor, A. M., da Silva Bessa, H. H., \& Barros, R. M. S. (2017). A utilização de plantas medicinais e fitoterápicos em prol da saúde. Única cadernos acadêmicos, 3(1). 